\section{Feigenblatt oder Katalysator?}

\section{Der in der Finanzwelt angesehene Hochschullehrer Henner Schierenbeck schrieb 1992 gemeinsam mit Eberhard Seidel über die Bedeutung von alternativen Ban- ken: „Ihre eigentliche Funktion liegt darin, als 'Katalysator des Wandels' zu wirken. Öko-Banken bieten Anregungen, fungieren in der Rolle des Vorreiters und Pioniers. Auf diese Weise können sie die ökologische Orientierung der Uni- versalbanken beschleunigen" (1). Sieben Jahre später lohnt es zu fragen, inwiefern die alternativen Banken diese Funktion erfüllen.}

$\mathrm{H}$ auptanliegen der ,grünen“ Banken ist die Produktökologie, also der verantwortungsvolle Umgang mit Geld. Bei gewöhnlichen Kreditinstituten finanziert der erbitterte Atomkraftgegner über seine Spareinlage im Ergebnis möglicherweise einen Atomkraftbetreiber oder -hersteller mit. Die alternativen Banken versuchen durch Zweckbindungen der Einlagen dieser Gefahr entgegenzuwirken. Entweder werden die Einlagen nur für definierte Projekte - beispielsweise Windparks - verwandt. Oder der Kreditnehmer und sein Projekt wird dem Einleger bekanntgegeben. Zusätzlich bieten einige der Ökobanken einen Zinsverzicht für den Einleger an. Die von der Bank gesparten Zinskosten werden an den Kreditnehmer mit einem entsprechend günstigeren Kreditzins weitergegeben. Kreditkunden, die aus zweckgebundenen Einlagen bedient werden, müssen eine umfassende Kreditprüfung auch nach ökologischen und sozialen Kriterien über sich ergehen lassen.

Gemessen an betriebswirtschaftlichen Kenngrößen sind die drei Ausnahmebanken klein und kaum ein ernsthafter ökonomischer Konkurrent für die anderen Geldhäuser. Die Bilanzsummen liegen bei 205 Millionen (UmweltBank), 265 Millionen (GLS Gemeinschaftsbank) und 326 Millionen Mark (Ökobank). Sie sind mit regional agierenden Kreditgenossenschaften oder Sparkassen vergleichbar. Folgerichtig schreibt die Ökobank in ihrem Geschäftsbericht 1997: „Mit den ökonomischen Möglichkeiten der Bank allein können wir viele Projekte nicht realisieren. ... Insbesondere bei kleinen und mittelständischen, nicht börsennotierten Unternehmen ..., die sich mit neuartigen Projekten im Ökologieund Sozialbereich beschäftigen, bedarf es Risikokapitals in Form von Beteiligungen, da der Erfolg nicht mit Sicherheit voraussehbar ist“.
Der geringe Zulauf $z u$ den alternativen Banken läßt vermuten, daß das Kriterium der Umweltverträglichkeit für Anleger keine besonders hohe Priorität genießt. Vielmehr spielen bei der Wahl der Bank und des Anlageproduktes andere Kriterien wie die Rendite oder die Qualität der Beratung eine größere Rolle. Es kann angesichts dieses Befundes nicht verwundern, daß gewöhnliche Banken in der Vergangenheit kaum Schritte zu einer Ökologisierung der Produktpalette unternommen haben. Vereinzelt angebotene Investmentfonds, die in Umwelttechnologiewerten anlegen, haben aufgrund der geringen Marktbreite und krisenhaften Entwicklungen der Branche starke Wertschwankungen hinnehmen müssen. So wurde der Hypo Umweltfonds, den der ehemalige Greenpeace-Chef Walter Homolka in seiner Zeit bei der Hypo-Bank entwickelte, Ende 1996 aufgrund einer nicht überzeugenden Performance geschlossen. Mittlerweile haben sich einige grüne Investmentfonds wieder gefangen und versprechen eine marktgerechte Rendite. Dies sollte nicht darüber hinwegtäuschen, daß die Anlagevolumina in diesen Produkten nach wie vor gering sind. Die nachhaltigsten Ökologisierungsbemühungen der Banken sind bei der Kreditprüfung zu verzeichnen. Aufgrund der verschärften Umweltgesetzgebung kann ein Firmenkunde durch eine behördliche Auflage zur Schadstoffreduzierung unter Vollzugsdruck geraten und kurzfristig vor der Notwendigkeit nicht geplanter Investitionsausgaben stehen. Ferner kann er durch erhebliche Umweltabgaben oder Bußgeldzahlungen in seiner Ertragslage geschwächt werden. Dadurch steigt das Termin- oder Ausfallrisiko, die Bank muß befuirchten, daß sie das ausgeliehene Geld nicht zurückerhält. Kreditinstitute versuchen, diese Wagnisse in ihrer Kreditprüfung aufzuspüren. Dabei stehen sie vor dem besonderen Problem der Informationsbeschaffung. Aus den Jahresab- schlüssen sind selten Hinweise auf umweltschutzrelevante Maßnahmen zu entnehmen. Die Bankpraxis behilft sich mit Checklisten, um drohende Umweltgefahren zu erkennen. Auch diese Maßnahme des Risikomanagements dürtte kaum auf die Katalysatorenfunktion der alternativen Banken zurückgehen. Zusammenfassend gilt daher: Im Bereich der Produktökologie ist im Bankwesen wenig Engagement zu verzeichnen.

\section{Begrenzte Ausstrahlung}

Was bleibt von der optimistischen Einschätzung von Schierenbeck und Seidel übrig? Alternative Banken sind aufgrund ihrer Größe kaum geeignet, als 'Katalysator des Wandels' zu wirken. ÖkoBanken können Anregungen bieten. Zumindest bei der Kreditprüfung ähneln sich die Verfahren von alternativen Geldhäusern und den gewöhnlichen. Bei den ökologischen Anlageformen dürften die Umweltbanken Pate für deren Entwicklung gestanden haben. Nachhaltig erfolgreich sind diese Anlageprodukte bis heute nicht, sie sind ein Nischenprodukt geblieben. In der Zukunft wird es spannend zu beobachten sein, inwiefern von ökologisch akzentuierten Risikokapitalgesellschaften positive Impulse für ,,grüne“ Nischenanbieter ausgehen. Beispielsweise hat die Ökobank dazu Kontakte zur SG-Capital, Ökologik AG aufgenommen. Immerhin haben die alternativen Banken in der Öffentlichkeit ein Bewußtsein für den Zusammenhang von Geldgeschätten und Umweltproblematik geschaffen. Und damit sind Bankmanager für diese Fragen sensibilisiert. Ob diese Einsicht bei einer Finanzierungsentscheidung über ein ökologisch-problematisches Projekt handlungsleitend sein wird, dürtte aber von der Höhe der zu erwartenden Erlöse abhängen.

\section{Anmerkung}

(1) Schierenbeck, Henner/ Eberhard Seidel: Die Ökologisierung der Wirtschaft und das Bankgeschöft - Eine Einführung, in dies. (Hrsg.): Banken und Ökologie, Konzepte für die Umwelt, 1992.

\section{Der Autor}

Matthias Meifert ist als Berater bei der Kienbaum Management Consultants GmbH, Gummersbach tätig. Zuvor bekleidete er verschiedene Positionen in einem großem deutschen Kreditinstitut.

Kontakt: c/o Kienbaum Management Consultants, Ahlefelder Straße 47, 51645 Gummersbach, Tel. 02261/ 703-615, Fax -619, E-mail: matthias.meifer!@kienbaum.de 
(c) 20I0 Authors; licensee IÖW and oekom verlag. This is an article distributed under the terms of the Creative Commons Attribution Non-Commercial No Derivates License (http://creativecommons.org/licenses/by-nc-nd/3.o/), which permits unrestricted use, distribution, and reproduction in any medium, provided the original work is properly cited. 